\section{New Journals 1995}

CRITERIA for journals to be considered for review in this issue were circulated to publishers earlier this year, and were also published in Nature. They were that:

(1) the first number appeared during or after June 1993 and at least four separate numbers were issued by the end of May 1995 (although some of the journals eligible for, but not covered in, last year's review issue were also considered)*;

(2) the journal is published at least three times a year

(3) the main language used is English;

(4) where possible at least four issues should be made available for review, including the first and the most recent numbers.

The time criteria ensure that a reasonable sample of issues is available for judgement by the time reviews are commissioned.

Several journals known to satisfy the criteria were not submitted for review, or arrived too late for inclusion. It proved difficult to find reviewers for other, doubtless worthy journals, while some titles were considered to be of marginal interest to Nature's audience. Journals covering any aspect of science were

\section{Grant engineering}

\section{Richard Vile}

Gene Therapy. Editors Karol Sikora, Joseph Glorioso, Bob Williamson and Theodore Fridmann. Stockton. 6/yr. Europe $£ 145$, elsewhere $£ 155$ (institutional); $\$ 65$ (personal).

Cancer Gene Therapy. Editors Robert E. Sobel and Kevin J. Scanlon. Appleton and Lange. 4/yr. USA \$190, elsewhere \$215 (institutional); USA $\$ 85$, elsewhere $\$ 108$ (personal).

THE prospect of therapy is justification enough for researching the molecular genetics of just about any disease. As well as providing a warm glow of altruistic satisfaction, a very real advantage is that such research allows grants to be written with a distinctly therapeutic bent. So the advent of gene therapy into the clinic, albeit currently for drastic diseases and desperate patients, has led to an explosion in the number of research papers, reviews and clinical protocols - all of which must find a home in hard copy. These two journals are responses to this boom.

The journals share a similar structure, combining typically up to 6 or 7 original research papers with a general review, a literature survey, editorials and updates on clinical protocols that have been applied for or accepted. In addition, both journals cover annual meetings with publication of abstracts, providing a useful way to keep up to date with research ahead of the lag between submission of manuscripts and their appearance in print. For the most obvious difference one need look no further than the titles. Gene Therapy accepts eligible, although those dealing with clinical medicine and pure mathematics were excluded, as were abstracts publications. A list of eligible titles submitted for review but not covered appears on page 272 .

The brief given to the reviewers was to limit themselves to comments on the publications sent to them, and to avoid discussion of general questions of periodical publishing. Opinions expressed in the reviews are based on a sample of issues, usually the first, the most recent (as of the end of May) and two in between. As in previous years the preponderance of journals in the biological sciences reflects the bias of the material submitted.

Details of editors and frequency of publication, and the subscription rates appearing at the top of each review, are given in most instances for 1995. This information is not complete in all cases, and readers interested in subscribing to a particular journal should check the rate with the publisher concerned.

*See Nature 371, 440-458 (1994); 365, 569-589 (1993); 359, 435-464 (1992); 353, 457-481 (1991); 347, 581-599 (1990).

manuscripts covering advances relevant to any disease for which gene therapy holds out promise. So although cancer features strongly, other genetic diseases are also covered in both primary research and review articles. By contrast, Cancer Gene Therapy restricts its output more to papers directly on cancer its problems. Nevertheless, the reviews often deal with topics that have wide applicability in gene therapy as a whole (such as viral vectors for gene delivery) and there would be as strong a case for, say, cystic fibrosis therapists to subscribe as for molecular oncologists.

The broader appeal of these journals to sceptics of gene therapy, or researchers with less applied interests, is more uncertain. Both journals are concerned with papers directed essentially at therapists rather than on those reporting advances in our understanding of the molecular genetics of disease. On the other hand, genetherapy die-hards would argue that the therapeutic end of the molecular genetics ultimately provides its own justification. In addition, the problems associated with the delivery of genes to cure disease cover a wide range of disciplines, including virology, genetics and immunology. That both journals have now survived their first year, despite having to compete with the highly respected Human Gene Therapy, testifies to their acceptance by the gene-therapy community itself. It would seem prudent for most molecular geneticists to follow this trio of journals, if only to provide a little therapeutic boost to their grant applications.

Richard Vile is in the Richard Dimbleby Department of Cancer Research, St Thomas's Hospital, Lambeth Palace Road, London SE1 TEH, UK.

\section{Redox redux}

\author{
Joe M. McCord
}

Redox Report. Editors John W. Eaton, Nicholas H. Hunt and Simon P. Wolff. Churchill Livingstone. 4/yr. USA \$310, Europe $£ 200$, elsewhere $£ 202$ (institutional); USA \$155, Europe $£ 100$, else where $£ 102$ (personal).

REDOX Report focuses on free-radical research and oxidative processes as they apply to "biology, medicine, and all aspects of the human environment". At first glance one may question the need for another speciality journal in the relatively small (but still growing) arena of freeradical biology. Its competition is supplied by Free Radical Biology and Medicine and Free Radical Research and perhaps by Archives of Biochemistry and Biophysics, which is less specialized but increasingly somewhere to look for research in the field. Of the 1,380 papers published in 1994 on the subjects of superoxide or superoxide dismutase, I was surprised to find that only about 6 per cent of the papers appeared in these three journals. There seems to be more than enough activity to support a new publication.

What does Redox Report have to offer? The editorial policy stated in the inaugural issue is both thoughtful and provocative, and is recommended reading for all editors of peer-reviewed journals. Effort will be made, we are told, to choose reviewers who are early- to mid-career scientists - those who still have the time and inclination to be at the bench. Effort will also be made to ensure that papers on a particular subject or by particular authors do not always go to the same referee. This is a problem that has concerned me as an editor, reviewer and author, but it seems to be a matter for which few journals have a formal policy. It is crucial in a rapidly evolving specialized area that the biases and prejudices of a handful of pioneers should not be allowed to dominate the area, regardless of how valuable their own contributions may have been.

The first four issues offer papers covering the same interesting array of topics that one might expect from Free Radical Biology and Medicine. In addition to the preponderance of research articles, each issue so far has included some sort of paired point-counterpoint articles, illustrating the editors' determination to provide a forum for examining issues from all sides. Hypothesis articles are encouraged, as one of the journal's proclaimed goals is to spark the genesis of new ideas in the field. Even though biomedical journals tend to be serious business, an editorial such as the one entitled "Sleepy radicals" proves that even editors can have a sense of humour.

The layout is attractive and accessible. 